\title{
(Olympic) Sports (Sports Coverage)
}

\section{AUTHOR}

Catharina Vögele, Markus Schäfer

\section{KEYWORDS}

sports coverage, news selection

\section{BRIEF DESCRIPTION}

The variable,(Olympic) sports' identifies (Olympic) sports that are the subject of communication.

\section{FIELD OF APPLICATION/THEORETICAL FOUNDATION} The variable can be used in all fields of sports communication, but is particularly interesting with regard to communication about the Olympic Games. It is based on the current list of Olympic sports (IOC, 2020a) and the list of sports recognized by the International Olympic Committee (IOC, 2020b).

\section{REFERENCES/COMBINATION WITH OTHER METHODS OF DATA COLLECTION}

The list on which the category is based can also be used in surveys. In this way, it is possible, for example, to investigate whether the perception of relevance of (certain) sports in the population correspond or differ from the relevance journalists attribute to (certain) sports by the extent of their reporting.

\section{EXAMPLE STUDY}

In a study by Vögele and Schäfer (2020) on the use of content analyses in sports communication, the category was used in a slightly adapted form. They coded sports that were the subject of the respective content analyses. In this way, it was possible to determine which sports are in the focus of sports communication research (and which are not; $\mathrm{RH}=1.0$ ).

\section{(Olympische) Sportarten}

In dieser Kategorie werden die Sportarten verschlüsselt, über die der Beitrag berichtet. Sind die Olympischen Spiele allgemein Gegenstand der Berichterstattung, sind die übergeordneten Ausprägungen „100“ (Sommerspiele) bzw. „200“ (Winterspiele) zu codieren. Werden im Beitrag keine Sportarten oder olympischen Sportereignisse thematisiert, wird „0“ codiert. Sonstige Sportarten werden mit „9999“verschlüsselt.

000 keine (olympische) Sportart oder olympischen Sportereignisse themati-

\begin{tabular}{ll}
\hline $\mathbf{1 0 0 0}$ & Olympische Sommerspiele \\
\hline 1010 & American Football \\
\hline 1020 & Automobilsport \\
\hline 1030 & Badminton \\
\hline 1040 & Bandy \\
\hline
\end{tabular}




\section{(Olympische) Sportarten}

\begin{tabular}{|c|c|}
\hline 1050 & Baseball/Softball \\
\hline 1060 & Basketball \\
\hline 1070 & Beach Volleyball \\
\hline 1080 & Bergsteigen \\
\hline 1090 & Billiard \\
\hline 1100 & Bogenschießen \\
\hline 1110 & Boules \\
\hline 1120 & Bowling \\
\hline 1130 & Boxen \\
\hline 1140 & Bridge \\
\hline 1150 & Cheerleading \\
\hline 1160 & Cricket \\
\hline 1170 & Fechten \\
\hline 1180 & Floorball \\
\hline 1190 & Flugsport \\
\hline 1200 & Fußball \\
\hline 1210 & Frisbee \\
\hline 1220 & Gewichtheben \\
\hline 1230 & Golf \\
\hline 1240 & Handball \\
\hline 1250 & Hockey \\
\hline 1260 & Judo \\
\hline
\end{tabular}




\section{(Olympische) Sportarten}

\begin{tabular}{|c|c|}
\hline 1270 & Kanusport \\
\hline 1271 & Kanu Slalom \\
\hline 1272 & Kanu Sprint \\
\hline 1280 & Karate \\
\hline 1290 & Kickboxen \\
\hline 1300 & Korfball \\
\hline 1310 & Lacrosse \\
\hline 1320 & Leichtathletik \\
\hline 1330 & Moderner Fünfkampf \\
\hline 1340 & Motorbootsport \\
\hline 1350 & Motorradsport \\
\hline 1360 & Muaythai \\
\hline 1370 & Netball \\
\hline 1380 & Orientierungslauf \\
\hline 1390 & Pelota \\
\hline 1400 & Polo \\
\hline 1410 & Radsport \\
\hline 1411 & Bahnradsport \\
\hline 1412 & $B M X$ \\
\hline 1413 & Mountain Bike \\
\hline 1414 & Straßenradsport \\
\hline 1420 & Racquetball \\
\hline
\end{tabular}




\section{(Olympische) Sportarten}

\begin{tabular}{|c|c|}
\hline 1430 & Reitsport \\
\hline 1431 & Dressurreiten \\
\hline 1432 & Springreiten \\
\hline 1433 & Vielseitigkeitsreiten \\
\hline 1440 & Ringen \\
\hline 1441 & Freistilringen \\
\hline 1442 & Griechisch-römisches Ringen \\
\hline 1450 & Rudern \\
\hline 1460 & Rugby \\
\hline 1470 & Rollsport \\
\hline 1471 & Rollhockey \\
\hline 1472 & Rollkunstlauf \\
\hline 1473 & Inlineskaten \\
\hline 1474 & Inlinehockey \\
\hline 1480 & Sambo \\
\hline 1490 & Schach \\
\hline 1500 & Schießen \\
\hline 1510 & Schwimmsport \\
\hline 1511 & Freiwasserschwimmen \\
\hline 1512 & Schwimmen (Bahn) \\
\hline 1513 & Synchronschwimmen \\
\hline 1514 & Rettungsschwimmen \\
\hline
\end{tabular}




\section{(Olympische) Sportarten}

\begin{tabular}{|c|c|}
\hline 1520 & Segeln \\
\hline 1530 & Skateboarding \\
\hline 1540 & Sportklettern \\
\hline 1550 & Surfen \\
\hline 1560 & Taekwondo \\
\hline 1570 & Tanzen \\
\hline 1580 & Tauziehen \\
\hline 1590 & Tennis \\
\hline 1600 & Tischtennis \\
\hline 1610 & Trampolinsport \\
\hline 1620 & Turmspringen \\
\hline 1630 & Turnsport \\
\hline 1631 & Geräteturnen \\
\hline 1632 & Rhythmische Sportgymnastik \\
\hline 1640 & Triathlon \\
\hline 1650 & Squash \\
\hline 1660 & Sumoringen \\
\hline 1670 & Unterwassersport \\
\hline 1680 & Volleyball \\
\hline 1690 & Wasserball \\
\hline 1700 & Wasserski \\
\hline 1710 & Wushu \\
\hline
\end{tabular}




\section{(Olympische) Sportarten}

\begin{tabular}{|c|c|}
\hline 2000 & Olympische Winterspiele \\
\hline 2010 & Biathlon \\
\hline 2020 & Bobsport \\
\hline 2030 & Curling \\
\hline 2040 & Eishockey \\
\hline 2050 & Eiskunstlauf \\
\hline 2060 & Eisschnellauf \\
\hline 2070 & Eisstock \\
\hline 2080 & Nordische Kombination \\
\hline 2090 & Rodeln \\
\hline 2100 & Short Track \\
\hline 2110 & Skeleton \\
\hline 2120 & Ski Alpin \\
\hline 2130 & Skibergsteigen \\
\hline 2140 & Ski Freestyle \\
\hline 2150 & Skilanglauf \\
\hline 2160 & Skispringen \\
\hline 2170 & Snowboard \\
\hline 9999 & Sonstige Sportart \\
\hline
\end{tabular}

\section{REFERENCES}

Donsbach, W. (1987). Journalismusforschung in der Bundesrepublik. Offene Fragen trotz ,Forschungsboom'. In J. Wilke (Hrsg.), Zwischenbilanz der Journalistenausbildung (S. 105-142). München: Ölschläger.
Weischenberg, S. (1992). Journalistik. Theorie und Praxis aktueller Medienkommunikation. Band 1. Opladen: Westdeutscher.

Weston, M., Drust, B., Atkinson, G., \& Gregson, W. (2011). Variability of soccer referees match performances. International Jour- 
nal of Sports Medicine, 32(3), 190-194.

Vögele, C., \& Schäfer, M. (2019). Fußball-

Schiedsrichter im Spiegel der Medien:

Die Berichterstattung über Bundesliga-

Schiedsrichter in der ARD-Sportschau.

Journal für Sportkommunikation und

Mediensport, 4(1), 1-24. Verfügbar un-

ter: https://openjournals.hs-hannover.

de/jskms/article/view/111/96Text 